\title{
Is Serum Lactate Dehydrogenase Useful for Predicting Oncological Outcome in Patients With Soft Tissue Sarcoma?
}

\author{
TOMOKI NAKAMURA, KUNIHIRO ASANUMA, TOMOHITO HAGI and AKIHIRO SUDO
}

Department of Orthopaedic Surgery, Mie University Graduate School of Medicine, Tsu, Japan

\begin{abstract}
Background/Aim: The aim of the present study was to determine whether serum lactate dehydrogenase $(L D H)$ levels before treatment could predict the diseasespecific and event-free survival in adult patients with STS. Patients and Methods: A total of 142 adult patients with primary STS were reviewed. The average follow-up was 73 months with a minimum 2-year follow-up. Results: Age was significantly associated with LDH. Tumor grade, sex, tumor size, tumor depth, CRP levels, Hb levels and albumin levels were not related. $L D H$ levels were not significantly related to the oncological outcome including disease-specific survival and event-free survival in multivariate analysis. CRP levels were significantly related to the oncological outcome including event-free survival. Conclusion: Elevated $L D H$ levels prior to initial treatment were not found to be a poor prognostic factor of oncological outcome in adult STS, in both the univariate and multivariate analyses. We recommend routine measurement of the CRP levels for predicting oncological outcome.
\end{abstract}

The prognosis for patients with soft tissue sarcomas (STSs) has shown marked improvement over the past forth decades, however, those who develop local recurrence or metastatic disease continue to have high mortality rates. Especially, patients with high grade STS are at significant risk of relapse, and as many as 50\% of these patients die of their disease (1). Therefore, easily determinable, widely applicable and low-cost markers might help to improve the predictive ability for identifying a high risk for tumor relapse. As a reliable marker, elevated pretreatment CRP levels could serve as an independent risk factor for poor disease-specific survival and disease-free survival in patients with STS (2-4).

Correspondence to: Tomoki Nakamura, Department of Orthopaedic Surgery, Mie University Graduate School of Medicine. 2-174 Edobashi, Tsu-city, Mie, 514-8507, Japan. Tel: +81 592315022, Fax: +81592315211, e-mail: tomoki66@clin.medic.mie-u.ac.jp

Key Words: Lactate dehydrogenase, C-reactive protein, soft tissue sarcoma.
Lactate dehydrogenase (LDH) is a pyridine-linked enzyme which catalyzes the reduction of free pyruvate to lactate during glycolysis, as well as the oxidation of L-lactate to pyruvate during gluconeogenesis (5). Cancer cells have been found to utilize from five to ten times as much glucose as normal tissues do, converting most of it to lactate (5). Therefore, serum LDH concentration was found to have a prognostic role in many solid cancers, such as renal cell carcinoma, osteosarcoma, and lung cancer (6-8). However, the clinical significance of serum LDH levels in patients with STS has not been established. Therefore, we hypothesized that the levels of LDH may affect survival. The aim of the present study was to determine whether serum LDH before treatment could predict the disease-specific and event-free survival in adult patients with STS.

\section{Patients and Methods}

A total of 142 adult patients with primary STS who were treated between January 2005 and December 2014 were retrospectively reviewed. The histological diagnosis was confirmed in all patients. Patients that presented with recurrent disease, distant metastasis at diagnosis or who were referred for additional resection after a previous inappropriate excision, were excluded from this study. Among the 142 patients in the cohort, the average follow-up was 73 months (range=3.6-160 months) with a minimum 2-year followup. All patients underwent pretreatment staging with a lung computed tomography (CT) scan to rule out the presence of metastases. The histopathological diagnosis and tumor grade were determined for all patients by pathologists using the French Federation of Cancer Centers Sarcoma Group (FNCLCC) grading system (7). The LDH (standard level; 124-222 U/1), albumin (standard level; 4.1-5.1 g/dl) and CRP (0.3 mg/dl) levels were obtained prior to treatment for all patients, and were measured using mainly a Denka Seiken (Tokyo, Japan) X-2 autoanalyzer as part of a routine biochemical examination. A clinicopathological analysis was performed comparing the LDH levels to various factors including the age, gender, tumor size, tumor site, tumor depth and tumor histological grade.

The primary purpose of this study was to examine the prognostic factors, including LDH, associated with the patients' survival using univariate and multivariate analyses. The further aims were to examine the prognostic factors associated with the development of metastasis. 
Table I. The relationship between LDH and clinical characteristics.

\begin{tabular}{lccc}
\hline Variables & $\mathrm{n}$ & $\begin{array}{c}\text { LDH levels } \\
\text { (IU/1;mean) }\end{array}$ & $p$-Value \\
\hline Gender & & & \\
$\quad$ Male & 80 & 190 & 0.38 \\
$\quad$ Female & 62 & 196 & \\
Depth & & & \\
$\quad$ Superficial & 29 & 194 & 0.45 \\
$\quad$ Deep & 113 & 190 & \\
Tumor grade & & & \\
1 & 50 & 186 & 0.14 \\
2 & 31 & 186 & \\
3 & 61 & 202 & \\
\hline
\end{tabular}

LDH: Lactate dehydrogenase.

Statistical analysis. The statistical associations between the clinicopathological factors and LDH levels were evaluated using the Mann-Whitney $U$-test and Kruskal-Wallis test for quantitative data, and the $\chi^{2}$ test for qualitative data. Correlations between continuous factors were tested using Spearman rank correlation analysis. The disease-specific survival (DSS) time was taken from the date of the initial treatment for the primary tumor to the date when the patient was documented to be alive or the date when the patient died due to sarcoma. The event-free survival (EFS) time was taken from the date of the initial treatment for the primary tumor to the date when the patient was documented to be relapsed (local recurrence or metastasis). The survival and event-free curves were constructed using the Kaplan-Meier method. The log-rank test was used to compare the survival and events in the patients. A multivariate analysis was performed using a Cox proportional hazard model. The variables included in the multivariate analysis were the significant factors identified in the univariate analysis. A value of $p<0.05$ was considered to be significant in all statistical analyses.

\section{Results}

Patient, tumor and treatment characteristics. The mean and median age of the patients at diagnosis was 62 years (range $=21-92$ years). There were 80 male and 62 female patients. The mean and median tumor size at diagnosis was $8.6 \mathrm{~cm}$ and $8 \mathrm{~cm}$ (range $=2-30 \mathrm{~cm}$ ), respectively. Sixty-one patients had grade 3 sarcomas, 31 had grade 2 and 50 had grade 1 tumors according to FNCLCC grading system.

The tumors were histologically classified as follows: 53 liposarcomas (35 well-differentiated type, 10 myxoid type, 7 de-differentiated type and one pleomorphic type), 23 undifferentiated pleomorphic sarcomas (UPS), 23 myxofibrosarcomas, 17 leiomyosarcomas, 7 malignant peripheral nerve sheath tumors (MPNST), 19 other tumors. The primary tumor sites were the thigh $(n=57)$, leg $(n=14)$, buttock $(n=14)$, back $(n=10)$, upper arm $(n=10)$, chest wall $(n=7)$, shoulder $(n=5)$ and other sites $(n=25)$, including two retroperitoneal lesions. The tumor depth was superficial in 29 patients and deep in 113 patients. Nine patients received
Table II. Correlation of LDH levels with clinical variables.

\begin{tabular}{lcl}
\hline Variables & Spearman $\varrho$ & $p$-Value \\
\hline Elevated CRP & 0.044 & 0.6 \\
Increasing age & 0.311 & 0.0002 \\
Larger tumor size & 0.039 & 0.64 \\
Elevated albumin & 0.014 & 0.87 \\
Elevated Hb & -0.037 & 0.66 \\
\hline
\end{tabular}

CRP: C-reactive protein; LDH: lactate dehydrogenase; $\mathrm{Hb}$ : haemoglobin.

adjuvant radiotherapy and 23 patients received adjuvant chemotherapy (17 preoperatively, 16 postoperatively). The LDH, CRP, hemoglobin (Hb) and albumin levels were measured before chemotherapy in these patients.

The relationship between the $L D H$ levels and clinical characteristics. The median level of LDH was $187.5 \mathrm{U} / 1$. The relationships between the clinicopathological features and the LDH levels are shown in Table I and II. Age was significantly associated with LDH. Tumor grade, sex, tumor size, tumor depth, CRP levels, Hb levels and albumin levels were not related.

Overall disease-specific survival and predictors of mortality (Tables III and IV). At the final follow-up, ninety-nine patients $(99 / 142 ; 69.7 \%)$ were alive, but 35 had died of disease and 8 had died of other causes. The disease-specific survival was $77.7 \%$ at five years. When we divided the patients into two groups according to the levels of $\mathrm{LDH}$, there was no significant difference between those with higher and those with lower levels of LDH. The 5-years disease-specific survival in patient with higher $(n=71)$ and lower levels of $\mathrm{LDH}(\mathrm{n}=71)$ was $73.6 \%$ and $81.8 \%$, respectively $(p=0.21)$.

A Cox proportional univariate analysis of all possible prognostic factors confirmed the predictive value of the albumin levels $(p=0.0003)$, Hb levels $(p=0.039), \mathrm{LDH}$ $(p=0.049)$, age $(p=0.006)$, CRP levels $(p<0.0001)$, tumor size $(p=0.02)$ and histological grade (grade $2: p=0.04$, grade 1: $p=0.001)$. Age $(p=0.02)$, tumor grade (grade $1: p=0.001$ $v s$. grade 3$)$ and tumor size $(p=0.0002)$ maintained their prognostic significance in the multivariate analysis. Tumor grade (grade2: $p=0.06 v s$. grade 3$), \mathrm{CRP}(p=0.07)$ and $\mathrm{Hb}$ levels $(p=0.07)$ had marginal significance.

When we excluded STS with grade 1 from the analysis, 92 patients with high-grade STS remained. The diseasespecific survival was $66.6 \%$ at five years. Of the 92 patients, 35 had died of disease at the 5-year follow-up. A Cox proportional univariate analysis of all possible prognostic factors confirmed the predictive value of the albumin levels $(p=0.0004)$, Hb levels $(p=0.005)$, CRP levels $(p<0.0001)$, 
Table III. The Cox proportional analysis for disease-specific survival in 142 patients.

\begin{tabular}{|c|c|c|c|c|c|c|c|}
\hline \multirow[t]{2}{*}{ Variables } & & \multicolumn{3}{|c|}{ Univariate analysis } & \multicolumn{3}{|c|}{ Multivariate analysis } \\
\hline & & HR & $95 \% \mathrm{CI}$ & $p$-Value & HR & $95 \% \mathrm{CI}$ & $p$-Value \\
\hline Age & Years & 1.04 & $1.011-1.07$ & 0.006 & 1.036 & $1.006-1.066$ & 0.02 \\
\hline \multirow[t]{2}{*}{ Gender } & Male & 1 & & & & & \\
\hline & Female & 1.435 & $0.725-2.841$ & 0.3 & & & \\
\hline \multirow[t]{2}{*}{ Tumor depth } & Subcutaneous & 1 & & & & & \\
\hline & Deep & 2.063 & $0.724-5.879$ & 0.18 & & & \\
\hline Tumor size & $\mathrm{cm}$ & 1.059 & 1.011-1.109 & 0.02 & 1.131 & $1.059-1.208$ & 0.0006 \\
\hline \multirow[t]{3}{*}{ Tumor grade } & 3 & 1 & & & & & \\
\hline & 2 & 0.386 & $0.139-0.939$ & 0.04 & 0.409 & $0.162-1.031$ & 0.06 \\
\hline & 1 & 0.036 & $0.005-0.263$ & 0.001 & 0.023 & $0.003-0.19$ & 0.004 \\
\hline Alb & $\mathrm{g} / \mathrm{dl}$ & 0.282 & $0.143-0.558$ & 0.0003 & 1.815 & $0.576-5.722$ & 0.31 \\
\hline $\mathrm{Hb}$ & $\mathrm{g} / \mathrm{dl}$ & 0.754 & $0.623-0.913$ & 0.04 & 0.804 & $0.634-1.021$ & 0.07 \\
\hline LDH & IU/1 & 1.008 & $1-1.017$ & 0.049 & 1.003 & $0.994-1.011$ & 0.56 \\
\hline CRP & $\mathrm{mg} / \mathrm{dl}$ & 1.211 & $1.125-1.302$ & $<0.0001$ & 1.103 & $0.991-1.227$ & 0.07 \\
\hline
\end{tabular}

Alb: Albumin; Hb: haemoglobin; LDH: lactate dehydrogenase; CRP: C-reactive protein; HR: hazard ratio; CI: confidence interval.

Table IV. The Cox proportional analysis for disease-specific survival in 92 patients with high-grade STS.

\begin{tabular}{|c|c|c|c|c|c|c|c|}
\hline \multirow[t]{2}{*}{ Variables } & & \multicolumn{3}{|c|}{ Univariate analysis } & \multicolumn{3}{|c|}{ Multivariate analysis } \\
\hline & & HR & $95 \% \mathrm{CI}$ & $p$-Value & HR & $95 \% \mathrm{CI}$ & $p$-Value \\
\hline Age & Years & 1.026 & $0.999-1.054$ & 0.058 & & & \\
\hline \multirow[t]{2}{*}{ Gender } & Male & 1 & & & & & \\
\hline & Female & 2.037 & $1.018-4.078$ & 0.04 & 1.863 & $0.64-5.38$ & 0.25 \\
\hline \multirow[t]{2}{*}{ Tumor depth } & Subcutaneous & 1 & & & & & \\
\hline & Deep & 1.545 & $0.541-4.411$ & 0.42 & & & \\
\hline Tumor size & $\mathrm{cm}$ & 1.1 & $1.042-1.162$ & 0.0006 & 1.11 & $1.039-1.187$ & 0.002 \\
\hline \multirow[t]{2}{*}{ Tumor grade } & 3 & 1 & & & & & \\
\hline & 2 & 0.388 & $0.159-0.943$ & 0.04 & 0.43 & $0.174-1.002$ & 0.43 \\
\hline Alb & $\mathrm{g} / \mathrm{dl}$ & 0.366 & $0.184-0.728$ & 0.37 & & & \\
\hline $\mathrm{Hb}$ & $\mathrm{g} / \mathrm{dl}$ & 0.774 & $0.648-0.925$ & 0.005 & 0.923 & $0.652-1.306$ & 0.65 \\
\hline LDH & $\mathrm{IU} / 1$ & 1.007 & $0.999-1.015$ & 0.11 & & & \\
\hline CRP & $\mathrm{mg} / \mathrm{dl}$ & 1.167 & $1.082-1.259$ & $<0.0001$ & 1.093 & $0.979-1.22$ & 0.11 \\
\hline
\end{tabular}

Alb: Albumin; Hb: haemoglobin; LDH: lactate dehydrogenase; CRP: C-reactive protein; HR: hazard ratio; CI: confidence interval.

tumor size $(p=0.0006)$, sex $(p=0.04)$ and histological grade $(p=0.04)$. Tumor size $(p=0.002)$ maintained its prognostic significance in the multivariate analysis.

Event-free rate and predictors of events (Tables V and VI). Thirty-one of the 142 patients $(22 \%)$ developed local tumor recurrence. Four of these 31 patients developed local recurrence after metastases. Forty of the 142 patients $(28 \%)$ developed metastases. Fifty-five patients developed local recurrence and/or metastasis. The five-year event-free rate was $63.8 \%$.

The Cox proportional univariate analysis revealed that lower albumin levels $(p=0.01)$, higher levels of CRP $(p<0.0001)$, tumor size $(p=0.002)$, higher age $(p=0.04)$, deep tumor location $(p=0.04)$ and histological high-grade tumors (grade 1: $p<0.0001$, grade 2: $p=0.02$ ) are significant predictors of occurrence of events in STS patients. A multivariate analysis showed that the albumin levels $(p=0.04)$, CRP levels $(p<0.0001)$, tumor size $(p=0.0006)$, age $(p=0.04)$ and tumor grade (grade1: $p<0.0001$, grade2: $p=0.03$ ) remained independent predictors of oncological events.

As mentioned above, when grade 1 STS was excluded from the analysis, 92 patients with high-grade STS remained. A Cox proportional univariate analysis of all possible prognostic factors confirmed the predictive value of CRP levels $(p<0.0001)$, tumor size $(p=0.002)$ and histological grade $(p=0.02)$. CRP levels $(p<0.0001)$ and tumor size $(p=0.02)$ maintained their prognostic significance in the multivariate analysis. 
Table V. The Cox proportional analysis for event-free survival in 142 patients.

\begin{tabular}{|c|c|c|c|c|c|c|c|}
\hline \multirow[t]{2}{*}{ Variables } & & \multicolumn{3}{|c|}{ Univariate analysis } & \multicolumn{3}{|c|}{ Multivariate analysis } \\
\hline & & HR & $95 \% \mathrm{CI}$ & $p$-Value & HR & $95 \% \mathrm{CI}$ & $p$-Value \\
\hline Age & Years & 1.021 & $1.001-1.04$ & 0.04 & 1.021 & $1.001-1.042$ & 0.04 \\
\hline \multirow[t]{2}{*}{ Gender } & Male & 1 & & & & & \\
\hline & Female & 1.014 & $0.595-1.729$ & 0.96 & & & \\
\hline \multirow[t]{2}{*}{ Tumor depth } & Subcutaneous & 1 & & & 1 & & \\
\hline & Deep & 2.282 & $1.027-5.071$ & 0.04 & 1.465 & $0.606-3.544$ & 0.4 \\
\hline Tumor size & $\mathrm{cm}$ & 1.062 & $1.022-1.104$ & 0.002 & 1.302 & $1.158-1.464$ & 0.0006 \\
\hline \multirow[t]{3}{*}{ Tumor grade } & 3 & 1 & & & & & \\
\hline & 2 & 0.445 & $0.227-0.872$ & 0.02 & 0.462 & $0.232-0.918$ & 0.03 \\
\hline & 1 & 0.121 & $0.051-0.287$ & $<0.0001$ & 0.108 & $0.043-0.267$ & $<0.0001$ \\
\hline Alb & $\mathrm{g} / \mathrm{dl}$ & 0.444 & $0.234-0.844$ & 0.01 & 2.238 & $1.045-4.791$ & 0.04 \\
\hline $\mathrm{Hb}$ & $\mathrm{g} / \mathrm{dl}$ & 0.91 & $0.772-1.074$ & 0.27 & & & \\
\hline LDH & IU/1 & 1.006 & $1-1.013$ & 0.07 & & & \\
\hline CRP & $\mathrm{mg} / \mathrm{dl}$ & 1.288 & $1.195-1.390$ & $<0.0001$ & 1.302 & $1.158-1.464$ & $<0.0001$ \\
\hline
\end{tabular}

Alb: Albumin; Hb: haemoglobin; LDH: lactate dehydrogenase; CRP: C-reactive protein; HR: hazard ratio; CI: confidence interval.

Table VI. The Cox proportional analysis for event-free survival in 92 patients with high-grade STS.

\begin{tabular}{|c|c|c|c|c|c|c|c|}
\hline \multirow[t]{2}{*}{ Variables } & & \multicolumn{3}{|c|}{ Univariate analysis } & \multicolumn{3}{|c|}{ Multivariate analysis } \\
\hline & & HR & $95 \% \mathrm{CI}$ & $p$-Value & HR & $95 \% \mathrm{CI}$ & $p$-Value \\
\hline Age & Years & 1.012 & $0.993-1.032$ & 0.21 & & & \\
\hline \multirow[t]{2}{*}{ Gender } & Male & 1 & & & & & \\
\hline & Female & 1.343 & $0.759-2.375$ & 0.31 & & & \\
\hline \multirow[t]{2}{*}{ Tumor depth } & Subcutaneous & 1 & & & 1 & & \\
\hline & Deep & 1.892 & $0.802-4.46$ & 0.15 & 1.465 & $0.606-3.544$ & 0.4 \\
\hline Tumor size & $\mathrm{cm}$ & 1.082 & $1.029-1.136$ & 0.002 & 1.302 & $1.158-1.464$ & 0.0006 \\
\hline \multirow[t]{2}{*}{ Tumor grade } & 3 & 1 & & & & & \\
\hline & 2 & 0.449 & $0.229-0.88$ & 0.02 & 0.508 & $0.257-1.002$ & 0.051 \\
\hline $\mathrm{Alb}$ & $\mathrm{g} / \mathrm{dl}$ & 0.583 & $0.299-1.137$ & 0.11 & & & \\
\hline $\mathrm{Hb}$ & $\mathrm{g} / \mathrm{dl}$ & 0.889 & $0.759-1.04$ & 0.14 & & & \\
\hline LDH & $\mathrm{IU} / \mathrm{L}$ & 1.005 & $0.998-1.011$ & 0.17 & & & \\
\hline CRP & $\mathrm{mg} / \mathrm{dl}$ & 1.231 & $1.139-1.331$ & $<0.0001$ & 1.195 & $1.102-1.125$ & 0.02 \\
\hline
\end{tabular}

Alb: Albumin; Hb: haemoglobin; LDH: lactate dehydrogenase; CRP: C-reactive protein. HR: hazard ratio; CI: confidence interval.

\section{Discussion}

Elevated LDH levels have been identified as a negative prognostic factor not only for hematological malignancies but also for solid tumors, such as renal cell carcinoma, lung cancer and osteosarcoma (6-10), although the detailed mechanism linking LDH to poor survival remains unknown. The current univariate and multivariate analyses showed that LDH was not associated with oncological outcome in 142 adult patients with STS. Although LDH was related with disease-specific survival in the univariate analysis, this was probably due to the effect of age. LDH was correlated to age and age was a prognostic factor in multivariate analysis. Furthermore, when we excluded STS with grade 1 from analysis, age and LDH were not prognostic factors for oncological outcome. No studies have so far shown the relationship between $\mathrm{LDH}$ and oncological outcome in adult STS patients based on a multivariate analysis. A previous study has also found no impact on the survival of 20 pediatric patients with rhabdomyosarcoma (11). Nishigaya et al. suggested that serum LDH levels might be elevated in uterine leiomyosarcoma, but identifying leiomyosarcoma by measurement of LDH alone was difficult (12). We only found one case report which suggested a positive relationship between LDH and oncologic outcome in pediatric patients with rhabdomyosarcoma, although only three cases were included (13). Therefore, we consider that the measurement of LDH may not contribute to predicting oncologic outcome in STS, although further examination in large cohort studies is necessary. 
We also found that CRP levels are associated with oncologic outcome. We have previously reported that CRP levels could be a useful prognostic marker in STS which was consistent with the present results (2). Recently, a meta-analysis suggested that elevated pretreatment CRP levels could serve as an independent risk factor for poor disease-specific survival and disease-free survival in patients with STS (3).

There are a few possible limitations associated with the present study. Although all patients had undergone pretreatment staging with chest $\mathrm{CT}$ scans and routine blood examinations to rule out the presence of other cancers, other chronic conditions which may increase LDH levels were not taken into consideration due to the lack of information. Furthermore, the timing of measurement may have affected the levels of $\mathrm{LDH}$, even if the measurement of LDH was obtained before treatment in all patients. Further large-scale prospective multicenter studies are now warranted to confirm and extend these findings in the present study. The retrospective nature of the study is another limitation. However, we consider that LDH could not be a useful prognostic marker in patients with STS.

\section{Conclusion}

Elevated LDH levels prior to initial treatment were not found to be a poor prognostic factor for oncological outcome in both the univariate and multivariate analyses for adult soft tissue sarcoma. We still recommend routine measurement of CRP levels for predicting oncological outcome in patients with STS.

\section{Conflicts of Interest}

The Authors declare that they have no conflicts of interest regarding this study.

\section{Authors' Contributions}

Tomoki Nakamura concepted the study design, collected data, performed the analyses, interpreted the results, and drafted the article. Tomohito Hagi, Kunihiro Asanuma collected and provided clinical input. Akihiro Sudo reviewed the manuscript.

\section{References}

1 Cormier JN and Pollock RE: Soft tissue sarcoma. CA Cancer J Clin 54(2): 94-109, 2004. PMID: 15061599. DOI: 10.3322/ canjclin.54.2.94

2 Nakamura T, Matsumine A, Matsubara T, Asanuma K, Uchida A and Sudo A: Clinical significance of pretreatment serum Creactive protein level in soft tissue sarcoma. Cancer 118(4): 1055-1061, 2012. PMID: 21761398. DOI: 10.1002/cncr. 26353

3 Wang W, Liu S, Fang E and Zhao X: The value of C-reactive protein as an independent prognostic indicator for diseasespecific survival in patients with soft tissue sarcoma: A metaanalysis. PloS One 14(7): e0219215, 2019. PMID: 31260491 DOI: 10.1371 journal.pone.0219215
4 Szkandera J, Gerger A, Liegl-Atzwanger B, Absenger G, Stotz M, Samonigg H, Maurer-Ertl W, Stojakovic T, Ploner F, Leithner A and Pichker M: Validation of the prognostic relevance of plasma C-reactive protein levels in soft-tissue sarcoma patients. Br J Cancer 109(9): 2316-2322, 2013. PMID: 24084772. DOI: 10.1038/bjc.2013.595

5 Gatenby RA and Gillies RJ: Why do cancers have high aerobic glycolysis? Nat Rev Cancer 4(11): 891-899, 2004. PMID: 15516961. DOI: $10.1038 / \mathrm{nrc} 1478$

6 Deng T, Zhang J, Meng Y, Zhou Y and Li W: Higher pretreatment lactate dehydrogenase concentration predicts worse overall survival in patients with lung cancer. Medicine (Baltimore) 97(38): e12524, 2018. PMID: 30235773. DOI: 10.1097/MD.0000000000012524

7 Chen J, Sun MX, Hua YQ and Cai ZD: Prognostic significance of serum lactate dehydrogenase level in osteosarcoma: a metaanalysis. J Cancer Res Clin Oncol 140(7): 1205-1210, 2014. PMID: 24682390. DOI: 10.1007/s00432-014-1644-0

8 Petrelli F, Cabiddu M, Coinu A, Borgonovo K, Ghilardi M, Lonati V and Barni S: Prognostic role of lactate dehydrogenase in solid tumors: a systematic review and meta-analysis of 76 studies. Acta Oncol 54(7): 961-970, 2015. PMID: 25984930. DOI: $10.3109 / 0284186 X .2015 .1043026$

9 Suguro M, Kanda Y, Yamamoto R, Chizuka A, Hamaki T, Matsuyama T, Takezako N, Miwa A and Togawa A: High serum lactate dehydrogenase level predicts short survival after vincristine-doxorubicin-dexamethasone (VAD) salvage for refractory multiple myeloma. Am J Hematol 65(2): 132-135, 2000. PMID: 10996830. DOI: 10.1002/1096-8652(200010) 65:2<132::aid-ajh7>3.0.co;2-1

10 Terpos E, Katodritou E, Roussou M, Pouli A, Michalis E, Delimpasi S, Parcharidou A, Kartasis Z, Zomas A, Symeonidis A, Viniou NA, Anagnostopoulos N, Economopoulos T, Zervas $\mathrm{K}$ and Dimopoloulos MA; Greek Myeloma Study Group, Greece: High serum lactate dehydrogenase adds prognostic value to the international myeloma staging system even in the era of novel agents. Eur J Haematol 85(2): 114-119, 2010. PMID: 20477863. DOI: 10.1111/j.1600-0609.2010.01466.x

11 Bien E, Rapala M, Krawczyk M and Balcerska A: The serum levels of soluble interleukin-2 receptor alpha and lactate dehydrogenase but not of B2-microglobulin correlate with selected clinic-pathological prognostic factors and response to therapy in childhood soft tissue sarcomas. J Cancer Res Clin Oncol 136(2): 293-305, 2010. PMID: 19693535. DOI: 10.1007/s00432-009-0661-X

12 Nishigaya Y, Kobayashi Y, Matsuzawa Y, Hasegawa M, Fukasawa I, Watanabe Y, Tokunaga H, Yaegashi N and Iwashita M: Diagnostic value of combination serum assay of lactate dehydrogenase. D-dimer, and C-reactive protein for uterin leiomyosarcoma. J Obstet Gynaecol Res 45(1): 189-194, 2019. PMID: 30152048. DOI: 10.1111/jog.13792

13 Moritake H, Kamimura S, Akiyoshi K, Nagatoshi Y, Chuman H and Okamura $\mathrm{J}$ : Prognostic significance of elevated lactate dehydrogenase and creatine kinase in patients with rhabdomyosarcoma. Med Periatr Oncol 40(3): 187-197, 2003. PMID: 12518348 . DOI: $10.1002 / \mathrm{mpo} .10115$

Received October 15, 2019

Revised October 28, 2019

Accepted October 29, 2019 